\title{
Le rapport entre changement et acquisition illustré au moyen du système du futur
}

\author{
Jan Lindschouw \& Stephanie Löbl (Université de Copenague, \\ Danemark)
}

\begin{abstract}
The future tense system in French has undergone important reorganisations during the period from Middle to Modern French. During this period the synthetic future (parlera 'will talk') has changed from a system in which it expressed two semantic values to a system in which it expresses one single value. At the same time, the analytic future (va parler 'is going to talk') has undergone the opposite evolution having thus expanded its domain of use and covers nowadays a part of the domain formerly belonging to the synthetic future. At the same time, it is interesting to observe a parallel between the change of the grammatical forms of the future tenses and their acquisition by learners of FLE. Indeed, the learners seem to master the future tense form in progression (i.e. the analytic future) earlier than the future tense form in the process of reduction (i.e. the synthetic future). Furthermore, they tend to use the analytic future as their default form.

In this contribution, three major factors are proposed as an explanation of this order of acquisition: 1 . The frequency of the future tense forms in the oral and written input that the students receive in and outside the classroom; 2 . The transfer from the students' native language (Danish) which only possesses an analytical future form (will + infinitive); 3. The relation between the form and the function of the two future tenses.

Finally, it is discussed to which extent the linguistic forms in the process of reduction should be taught.
\end{abstract}

Keywords: Synthetic future; analytic future; language change; language acquisition; French as a foreign language (FLE)

\section{Introduction}

Il est bien connu que le système du futur a subi des réorganisations considérables pendant la période qui va du moyen français au français moderne. Il est aussi connu que l'acquisition du système du futur pose problème à des apprenants scandinaves, dans la mesure où les langues scandinaves n'ont pas recours à différentes formes pour exprimer le futur, mais ne se servent que d'une seule forme analytique, contrairement aux langues romanes qui en possèdent au moins deux, une synthétique ou morphologique et une autre analytique ou périphrastique. Ces formes existent également dans une variante simple et une variante composée et expriment en outre des nuances modales selon le contexte (Lindschouw 2017, 401-405). 
Dans cette étude interdisciplinaire, nous nous proposons d'étudier deux domaines que l'on n'a pas l'habitude d'étudier ensemble. Premièrement, dans une perspective diachronique, nous allons examiner le changement du système du futur (c'est-à-dire l'alternance entre le futur dit synthétique (parlera) et le futur dit analytique (va parler)) pendant la période qui va du moyen français au français moderne; deuxièmement, sous un angle synchronique, nous allons étudier l'acquisition de ce même système par des apprenants danophones au niveau universitaire. Plus précisément, nous allons examiner si et dans quelle mesure les changements observés dans le système $\mathrm{du}$ futur se manifestent au niveau acquisitionnel. Enfin, nous allons étudier les facteurs conditionnant l'acquisition des formes de futur et leurs valeurs sémantiques respectives.

Il importe de souligner que l'analyse diachronique constitue l'arrière-plan de notre travail et le fondement de l'analyse acquisitionnelle. Les hypothèses qui nous permettent de combiner l'angle diachronique avec l'angle acquisitionnel sont les suivantes :

1. Le changement du système langagier français a un effet pour l'input que les apprenants du français langue étrangère reçoivent dans la classe et pendant leurs interactions éventuelles avec des locuteurs natifs.

2. Si les locuteurs natifs du français ont senti un besoin de simplifier le système du futur afin de rendre plus claire la communication, il faut supposer que les apprenant du français, qui ont d'ailleurs une maîtrise moins stable de cette langue que les locuteurs natifs, sentent le même besoin de simplification du système.

\section{Valeurs attribuées aux formes du futur}

Avant de répondre à nos questions de recherche, il importe de s'interroger sur les valeurs sémantiques que les deux formes du futur expriment. Il existe cependant des approches que l'on peut nommer non sémantiques, parce qu'elles considèrent ou bien que les deux futurs sont des variantes stylistiques dans certains contextes (Vet 1993, 80-83) ou bien qu'ils peuvent être neutralisés quand ils apparaissent dans les mêmes contextes linguistiques (Helland 1995, Poplack et Dion 2009, 574), par exemple après l'adverbe bientôt (1). Selon Helland $(1995,20)$, aucune explication pragmatique des deux futurs n'est pertinente dans ces contextes :

(1) Il va bientôt revenir / Il reviendra bientôt (Helland 1995, 20).

A part de telles approches non sémantiques, la plupart des chercheurs sont d'accord pour admettre qu'il faut attribuer une valeur sémantico-pragmatique aux deux formes du futur. Ainsi le futur analytique indiquerait une action immédiate ou proche, l'intentionnalité, le commencement d'une action, l'imminence, la conviction que l'action future se produira, un sens de quelque chose d'inévitable (Reid 1955, 37, Confais 1995), l'engagement du locuteur dans l'action future (Fleischman 1982), une action future préparée dans le présent (Vet 1993), un conditionnement actuel pour l'action future (Schrott 1997, 26-40, 2001, 160), un lien psychologique par rapport au moment de l'énonciation, un aspect prospectif (Fleischman 1982, Jeanjean 1988), et enfin une nuance subjective ou appréciative vis-à-vis de l'action future au moment de l'énonciation (Poplack et Turpin 1999, Larreya 2005, 350, Lansari 2009, 204). 
En revanche, le futur synthétique serait perçu comme neutre et psychologiquement détaché du moment de l'énonciation. Cette forme ne traduirait pas la conviction du locuteur que l'action future est en prolongation avec le moment de l'énonciation (Fleischman 1982, Confais 1995), mais par contre des actions futures qui ne sont pas préparées au moment de l'énonciation (Vet 1993) et par conséquent des actions futures virtuelles (Schrott 1997, 26-40, 2001, 160). La réalisation de l'action future peut être mise en doute, ce qui explique, d'après Confais $(1995,399)$ que seulement cette forme peut traduire une supposition portant sur le futur. En outre, le futur synthétique indiquerait de l'inférence et de l'imagination (Reid 1955), une rupture avec le présent, une conjecture, une injonction et une atténuation (Imbs 1968).

Poplack et Dion (2009, 565ss) observent que dans les grammaires modernes et historiques, il existe généralement un manque de consensus sur les valeurs fonctionnelles à attribuer aux deux formes du futur; elles considèrent même que certaines valeurs attribuées sont idiosyncrasiques. Nous constatons cependant que les définitions exposées ici se recouvrent largement puisqu'un grand nombre d'approches sont d'accord pour admettre que le futur analytique indique une action future préparée ou motivée par le moment de l'énonciation, alors que le futur synthétique introduit une action future détachée du moment de l'énonciation, idée que nous poursuivrons dans cette étude.

\section{Evolution du système du futur}

\subsection{Collecte des données}

Afin d'examiner la distribution des deux formes du futur d'un point de vue diachronique, nous avons collecté environ 2600 occurrences à partir de deux grands corpus diversifiés, Frantext et Dictionnaire du Moyen Français (DMF). 200 occurrences ont été collectées pour chaque forme verbale et chaque siècle. Ce nombre d'occurrences a été choisi afin de garantir une répartition de données égale à travers les siècles en question, dans la mesure où certaines formes du futur sont sous-représentées d'un siècle à l'autre dans les deux corpus consultés, notamment le futur analytique au début de la période examinée où il reste une innovation linguistique marginale. Afin d'assurer une certaine représentativité de notre corpus, les données ont été choisies, de façon à ce qu'elles représentent un vaste éventail de genres textuels (i.e. textes argumentatifs et narratifs, poésie, théâtre en vers et en prose, dialogues dans les textes narratifs littéraires, etc.) tout en reflétant l'oral représenté et l'écrit.

Puisque les valeurs sémantico-fonctionnelles des deux formes du futur nous intéressent, nous avons collecté 200 occurrences de chaque forme verbale par siècle. Un autre procédé aurait consisté en une collecte qui tiennent compte de la fréquence relative des deux formes du futur dans chaque siècle, ce qui aurait permis de se prononcer sur le rapport fréquentiel et distributionnel entre les deux formes. Cependant, ce procédé a été évité pour saisir les différentes valeurs des deux futurs, et non seulement leur fréquence mutuelle, et parce que les deux formes du futur sont largement sensibles à leur représentation à travers les différents genres textuels.

\section{$3.215^{e}$ siècle}

Le futur synthétique est attesté depuis le plus ancien français et se rencontre selon Wilmet $(1970,157)$ déjà dans Les Serments de Strasbourg de 842. Puisque nous nous 
intéressons à l'alternance entre les deux formes du futur, notre collecte de données commence à partir du moment où le futur analytique entre en scène, à savoir au cours du $15^{\mathrm{e}}$ siècle.

D'après Gougenheim (1929), Wilmet $(1970,191)$ et Fleischman $(1982,82$ ss), ce n'est qu'au $15^{\mathrm{e}}$ siècle que se produit une accélération décisive dans l'évolution du futur analytique, ce que confirment nos données, quoique les premières occurrences que nous avons observées datent du milieu du $14^{\mathrm{e}}$ siècle (1350). Il aurait été intéressant de prendre en considération également les données du $14^{\mathrm{e}}$ siècle, mais pour des questions d'espace, nous nous limitons au $15^{\mathrm{e}}$ siècle ici.

\begin{tabular}{|c|c|c|c|}
\hline \multicolumn{2}{|c|}{$\begin{array}{c}\text { Forme verbale } \\
\text { Valeur }\end{array}$} & \multirow[t]{2}{*}{ Futur synthétique } & \multirow[t]{2}{*}{ Futur analytique } \\
\hline Valeur générale & Valeur spécifique & & \\
\hline \multirow{3}{*}{ Temporelle } & $\begin{array}{l}\text { Sans rapport avec } \\
\text { l'énonciation }\end{array}$ & $121(60,5 \%)$ & $21(10,5 \%)$ \\
\hline & Rapport avec l'énonciation & $56(28,0 \%)$ & $4(2,0 \%)$ \\
\hline & Contexte historique & $2(1,0 \%)$ & $165(82,5 \%)$ \\
\hline \multirow{3}{*}{ Modale } & Épistémique & $0(0,0 \%)$ & $0(0,0 \%)$ \\
\hline & Déontique & $3(1,5 \%)$ & $9(4,5 \%)$ \\
\hline & Atténuation & $1(0,5 \%)$ & $0(0,0 \%)$ \\
\hline \multirow[t]{2}{*}{ Temporello-modale } & $\begin{array}{l}\text { Sans rapport avec } \\
\text { l'énonciation + nuance } \\
\text { épistémique }\end{array}$ & $12(6,0 \%)$ & $1(0,5 \%)$ \\
\hline & $\begin{array}{l}\text { Rapport avec l'énonciation } \\
\text { + nuance épistémique }\end{array}$ & $5(2,5 \%)$ & $0(0,0 \%)$ \\
\hline Inclassable & Inclassable & $0(0,0 \%)$ & $0(0,0 \%)$ \\
\hline \multicolumn{2}{|c|}{ Total: 400 occurrences } & 200 & 200 \\
\hline
\end{tabular}

Tableau 1 : Valeurs du futur au $15^{\mathrm{e}}$ siècle.

Comme il ressort de la tableau 1 , le futur synthétique au $15^{\mathrm{e}}$ siècle est employé en accord avec sa valeur de base (futur sans rapport avec le présent) dans la majorité des cas $(66,5 \%)^{1}$. Cet emploi est illustré en (2) où mengeront traduit une valeur future détachée du présent, parce que la subordonnée temporelle en quant marque un point de repère abstrait dans l'avenir :

(2) Ilz mengeront maint bon morseau / Les enffans, quant je seray vieulx. (VILLON, Le lais Villon et les poèmes variés, 23, 1456, cit. DMF).

\footnotetext{
${ }^{1}$ Ce chiffre est obtenu en additionnant ceux de la case intitulée temporelle avec celle intitulée temporellomodale.
} 
Cependant, le futur synthétique peut également indiquer une valeur de futur liée au moment de l'énonciation dans $30,5 \%$ des cas. Cet emploi est illustré sous (3), où l'adverbe à présent et le commentaire métadiscursif militent en faveur de cette interprétation :

(3) A present je ne parleray principalment que du tiers mirouer de humaine creature, et de la belle ymaige de la benoite Trinité (GERSON, Sermon pour la fête de la sainte trinité, 155, 1402, cit. DMF).

Cette distribution ne peut guère surprendre, puisqu'il faut supposer qu'avant l'apparition du futur analytique, la valeur du futur synthétique était bipartite, pouvant exprimer à la fois un futur sans rapport avec le moment de l'énonciation et ce que la nouvelle forme va exprimer, une valeur de futur liée au présent.

Pour ce qui est du futur analytique, force est de constater qu'il n'indique qu'une valeur de futur liée au moment de l'énonciation que dans 2,0\% des cas. En (4), vays accrocher n'indique pas un mouvement, mais fonctionne plutôt comme une périphrase, parce que l'âme en question est présente dans le contexte situationnel. L'impératif portarons du contexte motive la réalisation du futur analytique qui va se produire par la suite :

(4) SATHAN. Alons, Belzebuth, mauldit dyable, / Et portarons l'arme en enfer.

BELZEBUTH. Mes griffes sont toutes de fer, / De quoy je la vays accrocher. (ANONYME, La passion d'Auvergne, 112, 1477, cit. DMF).

Le futur analytique indique plus souvent une valeur de futur sans rapport avec le présent (11\%), comme sous (5), où nous avons affaire à un énoncé abstrait et générique. Cellui qui ne réfère pas à une personne déterminée, mais à une entité abstraite :

(5) Lors embrasse cellui qui lui rapporte / Et va passer ${ }^{2}$ trois fois devant la porte Pour veoir la preuve, [...] (CHARTIER, Le débat des deux fortunes d'amours, 163, 1412, cit. DMF).

Ce qui a suscité notre curiosité, c'est que le futur analytique exprime une valeur historique dans 82,5 \% des cas observés, observation qui rejoint celle de Barceló et Bres (2005, 168). Cet emploi, illustré sous (6), s'emploie notamment dans un contexte au passé comme une variante stylistique du passé simple (Togeby 1982, 386) et est presque exclusivement réservé aux récits dans la prose narrative littéraire. D'après Gougenheim $(1929,94)$, ce tour tire son origine de l'ancien gascon :

(6) Quant vint le dimenche à matin et jour qu'il trespassa, fist appeller devant lui tous ses barons, prelaz, son conseil et chancelier; adonc va parler devant eulz moult piteuses paroles, si que tous les contraigni à lermes. (PIZAN, Le livre des fais et bonnes meurs du sage roy Charles V, 185, 1404, cit. DMF).

\section{$3.318^{e}$ siècle}

Comme les changements survenus dans le système du futur sont relativement lents, nous n'examinerons pas la distribution des formes du futur dans tous les siècles du moyen français au français moderne, mais ferons des coupes synchroniques plus vastes afin de

\footnotetext{
${ }^{2}$ Nous n'interprétons pas aller comme un verbe de mouvement, mais comme un auxiliaire, dans la mesure où passer fonctionne déjà comme un verbe de mouvement.
} 
documenter l'évolution lente du système du futur. Si nous nous arrêtons à « mi-chemin », au $18^{\mathrm{e}}$ siècle, pendant le processus de changement du système du futur, nous constatons que la distribution des valeurs du futur synthétique reste plus ou moins la même qu'au $15^{\mathrm{e}}$ siècle. En revanche, les changements les plus considérables ont affecté le futur analytique. Au cours du $16^{\mathrm{e}}$ et $\mathrm{du} 17^{\mathrm{e}}$ siècle, son contenu stylistique et modal se réduit considérablement pour disparaître complètement au $18^{\mathrm{e}}$ siècle. En revanche, les occurrences où le futur analytique exprime un contenu futur lié au moment de l'énonciation s'élèvent jusqu'à 90,5\%. Cette valeur s'observe dans un vaste éventail de genres textuels (dans les textes argumentatifs et littéraires, de même que dans le discours direct et les pièces de théâtre), ce qui témoigne que le futur analytique employé avec cette valeur appartient à toute la réalité langagière.

\begin{tabular}{|c|c|c|c|}
\hline \multicolumn{2}{|c|}{ Valeur } & Futur synthétique & Futur analytique \\
\hline Valeur générale & Valeur spécifique & & \\
\hline \multirow{3}{*}{ Temporelle } & $\begin{array}{l}\text { Sans rapport avec } \\
\text { l'énonciation }\end{array}$ & $120(60,0 \%)$ & $17(8,5 \%)$ \\
\hline & Rapport avec l'énonciation & $51(25,5 \%)$ & $169(84,5 \%)$ \\
\hline & Contexte historique & $0(0,0 \%)$ & $0(0,0 \%)$ \\
\hline \multirow{3}{*}{ Modale } & Épistémique & $2(1,0 \%)$ & $0(0,0 \%)$ \\
\hline & Déontique & $1(0,5 \%)$ & $0(0,0 \%)$ \\
\hline & Atténuation & $0(0,0 \%)$ & $0(0,0 \%)$ \\
\hline \multirow[t]{2}{*}{ Temporello-modale } & $\begin{array}{l}\text { Sans rapport avec } \\
\text { l'énonciation + nuance } \\
\text { épistémique }\end{array}$ & $21(10,5 \%)$ & $0(0,0 \%)$ \\
\hline & $\begin{array}{l}\text { Rapport avec l'énonciation } \\
\text { + nuance épistémique }\end{array}$ & $5(2,5 \%)$ & $12(6,0 \%)$ \\
\hline Inclassable & Inclassable & $0(0,0 \%)$ & $2(1,0 \%)$ \\
\hline \multicolumn{2}{|c|}{ Total: 400 occurrences } & 200 & 200 \\
\hline
\end{tabular}

Tableau 2 : Valeurs du futur au $18^{\mathrm{e}}$ siècle.

\section{$3.420^{e} / 21^{e}$ siècles}

A l'état actuel de la langue, la distribution des valeurs du futur synthétique n'a pas énormément changé par rapport aux siècles précédents. Sa valeur préférée est toujours une action future détachée $\mathrm{du}$ moment de l'énonciation (environ $72 \%$ des occurrences). Cependant, sa capacité à exprimer un contenu futur lié au moment de l'énonciation a diminué petit à petit, de $28 \%$ ( $18^{\mathrm{e}}$ siècle $)$, à $18,5 \%\left(19^{\mathrm{e}}\right.$ siècle $)$ et à $19 \%$ $\left(20^{\mathrm{e}} / 21^{\mathrm{e}}\right.$ siècles). Le futur analytique connaît lui aussi un déclin pour ce qui est de sa capacité à exprimer une action future liée au présent (de $90,5 \%$ au $18^{\mathrm{e}}$ siècle à $74 \%$ au $20^{\mathrm{e}} / 21^{\mathrm{e}}$ siècle), mais ce déclin peut s'expliquer par le fait que sa valeur de futur détachée du présent augmente de $8,5 \%$ au $18^{\mathrm{e}}$ siècle à environ $22 \%$ au $20^{\mathrm{e}}$ et $21^{\mathrm{e}}$ siècles. 


\section{Forme verbale}

Valeur

Futur synthétique Futur analytique

\begin{tabular}{|c|c|c|c|}
\hline & & \\
\hline Valeur générale & Valeur spécifique & \multirow[b]{2}{*}{$135(61,4 \%)$} & \multirow[b]{2}{*}{$42(19,1 \%)$} \\
\hline \multirow{3}{*}{ Temporelle } & $\begin{array}{l}\text { Sans rapport avec } \\
\text { l'énonciation }\end{array}$ & & \\
\hline & Rapport avec l'énonciation & $38(17,3 \%)$ & $129(58,6 \%)$ \\
\hline & Contexte historique & $9(4,1 \%)$ & $1(0,5 \%)$ \\
\hline \multirow{3}{*}{ Modale } & Épistémique & $2(0,9 \%)$ & $0(0,0 \%)$ \\
\hline & Déontique & $3(1,4 \%)$ & $7(3,2 \%)$ \\
\hline & Atténuation & $4(1,8 \%)$ & $0(0,0 \%)$ \\
\hline \multirow{2}{*}{ Temporello-modale } & $\begin{array}{l}\text { Sans rapport avec } \\
\text { l'énonciation + nuance } \\
\text { épistémique }\end{array}$ & $25(11,4 \%)$ & $7(3,2 \%)$ \\
\hline & $\begin{array}{l}\text { Rapport avec l'énonciation } \\
\text { + nuance épistémique }\end{array}$ & $4(1,8 \%)$ & $34(15,5 \%)$ \\
\hline Inclassable & Inclassable & $0(0,0 \%)$ & $2(1,0 \%)$ \\
\hline \multicolumn{2}{|c|}{ Total: 440 occurrences } & 220 & 220 \\
\hline
\end{tabular}

Tableau 3 : Valeurs du futur au $20^{\mathrm{e}} / 21^{\mathrm{e}}$ siècles.

\subsection{Conclusions}

La partie diachronique de ce travail a montré la distribution et l'évolution du futur synthétique et du futur analytique au cours de la période qui va du moyen français au français moderne. Bien que ces deux formes aient chacune une valeur de base, le futur synthétique indiquant une valeur de futur détachée du moment de l'énonciation et le futur analytique véhiculant une valeur de futur intimement liée à ce point, le système a subi des changements.

Si le futur synthétique n'a pas subi de changements considérables, sauf que sa valeur bipartite originelle s'est réduite, le futur analytique, en revanche, a subi les changements les plus importants. Cette forme possédait en moyen français avant tout une valeur stylistique et modale, mais s'est transformée progressivement en une forme à valeur temporelle dont la fonction primaire est de traduire une valeur de futur en rapport avec le présent. Une fois que cette valeur temporelle s'est établie, le futur analytique est devenu petit à petit en mesure d'exprimer aussi une valeur de futur détachée du moment de l'énonciation. Le futur analytique est donc passé d'un système à valeur unitaire à un système à valeurs bipartites ${ }^{3}$.

\footnotetext{
${ }^{3}$ On peut certes analyser l'évolution du système du futur comme un processus de grammaticalisation, plus précisément comme un cas de régrammation (Andersen 2006, Nørgård-Sørensen, Heltoft et Schøsler 2011), mais cette discussion est hors de la portée du présent travail, mais poursuivie dans Lindschouw (2011).
} 


\section{Acquisition du système du future}

Le but de cette partie de notre contribution est d'examiner si les étudiants en français s'approprient les formes verbales analytiques avant les formes synthétiques. Le travail examine en outre l'ordre de l'acquisition en identifiant les facteurs qui influent sur l'acquisition des formes grammaticales afin de pouvoir améliorer l'enseignement de la grammaire dans le système éducatif danois.

Le domaine du futur est bien entendu grammaticalisé en français contrairement au danois. En danois, on utilise soit une construction avec un semi-auxiliaire + infinitif (7) soit le présent (8), mais qui ne traduit pas la même différence qu'on observe dans le système du français. La construction avec le semi-auxiliaire + infinitif ressemble structurellement à la forme analytique française.

(7) Vi skal gå nu 'Nous allons partir maintenant'.

(8) Om et par dage tager jeg hen for at besøge min far 'Dans quelques jours, je pars voir mon père'.

Il existe donc des différences remarquables dans ce domaine entre les deux langues. Ainsi, les apprenants du français langue étrangère sont confrontés à une difficulté de choix entre les formes du futur appropriées.

En outre, la forme analytique est souvent appelée le futur proche (Ayoun 2013, 17, Franckel 1984, 65, Hansén et Schwartz 1995, 170), ce qui n'est ni adéquat ni pédagogique, dans la mesure où le futur analytique n'indique pas nécessairement une valeur de futur imminente par rapport au présent, mais plutôt un événement préparé ou motivé par ce dernier (9). C'est pourquoi, nous utilisons le terme de futur composé pour éviter des malentendus potentiels.

(9) Tu as appris la nouvelle ? Marie va avoir un enfant.

Puisqu'il n'existe pas deux formes de futur parallèles en danois et que le système du futur a subi les réorganisations esquissées en section 3, ce qui veut dire que l'input que reçoivent les étudiants, soit oral (émissions télévisées, radio, interactions, etc.) soit écrit (littérature moderne), est censé contenir plus de formes analytiques que synthétiques, il est pertinent de poser les questions suivantes :

a. Les étudiants en français s'approprient-ils les formes verbales analytiques avant les formes synthétiques?

b. Les formes en voie de réduction sémantique sont-elles acquises plus tard et avec plus de difficulté que les formes en progression sémantique?

c. Dans, l'affirmative, pourquoi l'acquisition se passe-t-elle dans cet ordre et quels sont les facteurs qui influent sur l'acquisition des formes du futur?

\subsection{Méthologie}

Ces questions sont testées dans une étude d'effet dans laquelle nous examinons l'acquisition des catégories susmentionnées de 20 étudiants danois de l'université de Copenhague, divisés en deux groupes : un groupe expérimental qui reçoit des leçons ciblées 
sur les formes et fonctions des deux futurs, et un groupe de contrôle qui ne reçoit pas de leçons. Les deux groupes d'étudiants sont relativement homogènes avec un âge moyen de 26 ans.

L'enseignement que reçoit le groupe expérimental se focalise explicitement sur la sémantique et l'influence des adverbes temporels sur les formes du futur.

Cette partie de l'étude se compose de données empiriques issues de quatre tests. Les tests, tous adaptés selon les résultats d'un test-pilote, sont effectués à travers une période de deux mois. Les étudiants ont rempli un pré-test (test préliminaire) avant le commencement de l'enseignement ciblé, deux post-tests après chaque enseignement, et un post-test un mois après, afin de pouvoir mesurer les effets immédiats et ceux à long terme. Avant les post-tests, seul le groupe expérimental a reçu des leçons portant sur l'emploi du futur en français.

Dans les tests, nous avons inclus plusieurs exercices ouverts et fermés relatifs à leurs compétences réceptives et productives. Nous examinons également leurs connaissances déclaratives et procédurales.

\subsection{Résultats}

Dans les tableaux suivants, les formes grammaticales sont représentées par des abréviations : le futur analytique (FA) et le futur synthétique (FS).

Les résultats du prétest, concernant la compétence réceptive, montrent que les étudiants reconnaissent presque tous les deux formes. Un des exercices examine directement leur connaissances déclaratives en leur demandant de définir l'usage des formes. En général, les étudiants témoignent de connaissances superficielles de l'emploi des formes, et on observe également des malentendus. Par exemple, plusieurs étudiants décrivent la forme analytique comme un futur proche, près du présent.

Pour ce qui est de la compétence productive, les résultats montrent que les deux groupes ont tendance à favoriser la forme analytique au détriment du futur synthétique, bien qu'elle ne soit utilisée correctement que dans moins de $15 \%$ des cas :

\begin{tabular}{|c|c|c|c|c|c|c|c|c|c|}
\hline & $\begin{array}{l}\text { Tokens } \\
\text { FA }\end{array}$ & $\begin{array}{l}\text { Tokens } \\
\text { FS }\end{array}$ & $\begin{array}{l}\text { Types } \\
\text { FA }\end{array}$ & $\begin{array}{l}\text { Types } \\
\text { FS }\end{array}$ & $\begin{array}{l}\text { Tokens } \\
\text { FA } \\
\text { corrects }\end{array}$ & $\begin{array}{l}\text { Tokens } \\
\text { FS } \\
\text { corrects }\end{array}$ & $\begin{array}{l}\text { Tokens } \\
\text { d'autres } \\
\text { temps }\end{array}$ & $\begin{array}{l}\text { Faute } \\
\text { d'ortho. } \\
\text { FA }\end{array}$ & $\begin{array}{l}\text { Faute } \\
\text { d'ortho. } \\
\text { FS }\end{array}$ \\
\hline \multirow{3}{*}{$\begin{array}{l}\text { Groupe de } \\
\text { contrôle }\end{array}$} & 14 & 5 & \multirow{3}{*}{13} & \multirow{3}{*}{5} & 2 & 5 & 7 & \multirow{3}{*}{0} & \multirow{3}{*}{0} \\
\hline & $14 / 26=$ & $5 / 26=$ & & & $2 / 14=$ & $5 / 5=$ & $7 / 26=$ & & \\
\hline & $53,8 \%$ & $19,2 \%$ & & & $14,3 \%$ & $100 \%$ & $26,9 \%$ & & \\
\hline \multirow{3}{*}{$\begin{array}{l}\text { Groupe } \\
\text { expérimental }\end{array}$} & 19 & 11 & \multirow{3}{*}{17} & \multirow{3}{*}{11} & 1 & 11 & 11 & \multirow{3}{*}{0} & \multirow{3}{*}{1} \\
\hline & $19 / 41=$ & $11 / 41=$ & & & $1 / 19=$ & $11 / 11=$ & $11 / 41=$ & & \\
\hline & $46,3 \%$ & $26,8 \%$ & & & $5,3 \%$ & $100 \%$ & $26,8 \%$ & & \\
\hline
\end{tabular}

Tableau 4 : Résultat de l'exercice du pré-test en \%.

Il y a de nombreuses fautes dans des contextes avec certains adverbes temporels comme dans l'exemple (10) qui combine l'adverbe temporel La semaine prochaine avec la forme analytique : 


\section{(10) Stud3t;6,2: La semaine prochaine, je vais faire mes études.}

En outre, les étudiants utilisent d'autres temps verbaux, notamment le présent, dans plus de $20 \%$ des cas, alors que le futur synthétique est quasiment absent.

En ce qui concerne la compétence réceptive dans le post-test, les deux groupes reconnaissent les deux formes. En outre, les données montrent que seuls les étudiants du groupe de contrôle continuent à utiliser le terme de futur proche pour décrire la forme analytique. Enfin, le groupe expérimental décrit l'usage des forms de façon plus approfondie que dans le pré-test.

La tableau 5 montre qu'après l'enseignement, dans la production libre, le groupe expérimental utilise dans plus de $80 \%$ des cas le futur synthétique, et dans une large mesure correctement, alors que le groupe de contrôle continue à favoriser le futur analytique. De plus, le groupe expérimental ont amélioré leur emploi du futur analytique considérablement quoiqu'il ne le maitrise pas complètement.

\begin{tabular}{|c|c|c|c|c|c|c|c|c|c|}
\hline \multicolumn{10}{|c|}{ Qu'est-ce que vous ferez la semaine prochaine? } \\
\hline & $\begin{array}{l}\text { Tokens } \\
\text { FA }\end{array}$ & $\begin{array}{l}\text { Tokens } \\
\text { FS }\end{array}$ & $\begin{array}{l}\text { Types } \\
\text { FA }\end{array}$ & $\begin{array}{l}\text { Types } \\
\text { FS }\end{array}$ & $\begin{array}{l}\text { Tokens } \\
\text { FA } \\
\text { corrects }\end{array}$ & $\begin{array}{l}\text { Tokens } \\
\text { FS } \\
\text { corrects }\end{array}$ & $\begin{array}{l}\text { Tokens } \\
\text { d'autres } \\
\text { temps }\end{array}$ & $\begin{array}{l}\text { Faute } \\
\text { d'ortho. } \\
\text { FA }\end{array}$ & $\begin{array}{l}\text { Faute } \\
\text { d'ortho. } \\
\text { FS }\end{array}$ \\
\hline \multirow{3}{*}{$\begin{array}{l}\text { Groupe de } \\
\text { contrôle }\end{array}$} & 11 & 9 & \multirow{3}{*}{9} & \multirow{3}{*}{9} & 1 & 7 & 10 & \multirow{3}{*}{1} & \multirow{3}{*}{3} \\
\hline & $11 / 30=$ & $9 / 30=$ & & & $1 / 11=$ & $7 / 9=$ & $10 / 30=$ & & \\
\hline & $36,7 \%$ & $30 \%$ & & & $9,1 \%$ & $77,8 \%$ & $33,3 \%$ & & \\
\hline \multirow{3}{*}{$\begin{array}{l}\text { Groupe } \\
\text { expérimental }\end{array}$} & 7 & 27 & \multirow{3}{*}{7} & \multirow{3}{*}{25} & 2 & 27 & 4 & \multirow{3}{*}{0} & \multirow{3}{*}{3} \\
\hline & $7 / 32=$ & $27 / 32=$ & & & $2 / 7=$ & $27 / 27=$ & $4 / 32=$ & & \\
\hline & $21,9 \%$ & $84,4 \%$ & & & $28,6 \%$ & $100 \%$ & $12,5 \%$ & & \\
\hline
\end{tabular}

Tableau 5 : Résultat de l'exercice ouvert du post-test en \%.

De plus, les étudiants du groupe expérimental ont tendance à avoir moins recours à d'autres temps verbaux pour exprimer le futur que le groupe de contrôle. Leur production semble refléter leur connaissance déclarative. Le groupe de contrôle utilise toujours la forme analytique quel que soit le choix d'adverbe temporel (11), alors que le groupe expérimental combine correctement les adverbes temporels du futur avec la forme synthétique (12) :

(11) Stud7k;5: La semaine prochaine ma meilleure amie va me rendre visite.

(12) Stud2t;5: La semaine prochaine, je verrai ma copine Julie.

Le dernier post-test a été effectué un mois plus tard pour vérifier si les étudiants ont retenu les connaissances apprises dans le domaine du futur. Pour ce qui est de la compétence réceptive, nous n'observons pas de différence majeure entre les deux post-tests.

Dans la description des formes, en général, les groupes fournissent des définitions correctes des deux formes. Pourtant, le groupe expérimental témoigne d'une connaissance déclarative plus profonde et solide que le groupe de contrôle.

Quant à la compétence productive, nous constatons toujours des différences entre les productions des deux groupes. En répondant à la question Qu'est-ce que vous ferez dans une semaine ? qui contient un adverbe de futur, le groupe de contrôle a tendance à favoriser la 
forme analytique alors que le groupe expérimental utilise plutôt la forme synthétique, comme l'illustre le tableau 6 :

\begin{tabular}{|c|c|c|c|c|c|c|c|c|c|}
\hline & $\begin{array}{l}\text { Tokens } \\
\text { FA }\end{array}$ & $\begin{array}{l}\text { Tokens } \\
\text { FS }\end{array}$ & $\begin{array}{l}\text { Types } \\
\text { FA }\end{array}$ & $\begin{array}{l}\text { Types } \\
\text { FS }\end{array}$ & $\begin{array}{l}\text { Tokens } \\
\text { FA } \\
\text { corrects }\end{array}$ & $\begin{array}{l}\text { Tokens } \\
\text { FS } \\
\text { corrects }\end{array}$ & $\begin{array}{l}\text { Tokens } \\
\text { d'autres } \\
\text { temps }\end{array}$ & $\begin{array}{l}\text { Faute } \\
\text { d'ortho. } \\
\text { FA }\end{array}$ & $\begin{array}{l}\text { Faute } \\
\text { d'ortho. } \\
\text { FS }\end{array}$ \\
\hline \multirow{3}{*}{$\begin{array}{l}\text { Groupe de } \\
\text { contrôle }\end{array}$} & 10 & 4 & \multirow{3}{*}{10} & \multirow{3}{*}{4} & 3 & 4 & 6 & \multirow{3}{*}{2} & \multirow{3}{*}{0} \\
\hline & $10 / 20=$ & $4 / 20=$ & & & $3 / 10=$ & $4 / 4=$ & $6 / 20=$ & & \\
\hline & $50 \%$ & $20 \%$ & & & $30 \%$ & $100 \%$ & $30 \%$ & & \\
\hline \multirow{3}{*}{$\begin{array}{l}\text { Groupe } \\
\text { expérimental }\end{array}$} & 5 & 31 & \multirow{3}{*}{5} & \multirow{3}{*}{31} & 2 & 30 & 4 & \multirow{3}{*}{0} & \multirow{3}{*}{7} \\
\hline & $5 / 40=$ & $31 / 40=$ & & & $2 / 5=$ & $30 / 31=$ & $4 / 40=$ & & \\
\hline & $12,5 \%$ & $96,9 \%$ & & & $40 \%$ & $96,8 \%$ & $10 \%$ & & \\
\hline
\end{tabular}

Tableau 6 : Résultat de l'exercice ouvert du dernier post-test en \%.

\subsection{Conclusions}

La partie acquisitionnelle a montré que dans le pré-test, avant l'enseignement ciblé, les deux groupes favorisent la forme analytique comme la forme standard du futur, alors que, généralement, elle n'est utilisée correctement que dans moins de $15 \%$ des cas.

À travers la période examinée, le groupe expérimental s'améliore plus que le groupe de contrôle, notamment pour ce qui est de l'emploi de la forme synthétique, mais aussi de la forme analytique. En outre, il réduit l'emploi d'autres formes pour exprimer le futur, contrairement au groupe de contrôle.

Le groupe expérimental témoigne de l'amélioration la plus considérable pour ce qui est de l'emploi du futur synthétique, tant au niveau déclaratif que procédural. Les résultats indiquent par conséquent qu'une connaissance déclarative approfondie est utile pour améliorer les compétences procédurales.

\section{Conclusions et explications}

La première partie de cette étude a été consacrée aux changements survenus dans le système du futur. Au cours du temps, le futur synthétique a réduit son domaine d'emploi, alors que le futur analytique a élargi le sien, ce qui permet de considérer cette forme comme la forme non marquée ${ }^{4}$ pour exprimer le futur en français moderne, puisqu'il traduit non seulement une valeur de futur intimement liée au moment de l'énonciation, mais aussi la valeur autrefois réservée au futur synthétique, à savoir une valeur de futur coupée du moment de l'énonciation.

Ensuite, nous avons montré que l'acquisition du futur synthétique pose problème aux apprenants danophones, tandis qu'ils maîtrisent beaucoup mieux le futur analytique avant

\footnotetext{
${ }^{4}$ L'opposition de marquage entre deux formes concurrentielles à l'intérieur d'un paradigme clos peut être analysée au niveau morphologique, sémantique et textuel (i.e. fréquentiel) (Givón 1990, 947). Nous n'analysons ici l'opposition entre les deux formes de futur qu'en termes de marquage sémantique, puisque ce niveau est le plus décisif pour établir une opposition de marquage comme Andersen (2001) l'a clairement montré.
} 
l'enseignement ciblé. Cela confirme que les formes en voie de régression de la langue sont celles qui s'acquièrent plus tardivement que les formes en progression lors du processus d'acquisition des apprenants.

Cependant, la deuxième partie de l'étude montre que les apprenants améliorent leur emploi et leurs connaissances des formes du futur à travers un enseignement ciblé qui focalise entre autres sur l'emploi des adverbes temporels.

On peut s'interroger sur les facteurs censés determiner l'acquisition des deux formes du futur. On peut identifier trois types de facteurs, à savoir: la fréquence des formes, le transfert de la langue maternelle et les occurrences où la relation entre la forme et la fonction des éléments linguistiques est précise.

Premièrement, les formes qu'un apprenant de la langue cible rencontre fréquemment sont celles qui s'approprieront le plus rapidement et le plus aisément (Lund 2009). Le fait que la fréquence de la forme analytique ait augmenté alors que celle de la forme synthétique a décliné en français moderne, contrairement à des étapes révolues du français (voir section 3) semble influer sur l'acquisition du système du futur, dans la mesure où l'input écrit et oral que reçoivent les apprenants est censé contenir plus de formes du futur analytique que du futur synthétique.

Deuxièmement, le transfert de la langue danoise joue fort probablement aussi un rôle pour l'acquisition du système du futur français, puisque l'expression du futur en danois se forme à partir d'un verbe semi-auxiliaire skal + l'infinitif, ce qui ressemble à la forme analytique en français, bien que leurs contenus sémantiques ne soient pas identiques (Wiberg 2002).

Troisièmement, l'analyse montre que les étudiants du groupe expérimental font plus de progrès dans les cas où un adverbe temporel est présent dans le contexte et où sa signification sémantique est claire et visible. Dans ces cas-là, le rapport entre la forme et la fonction du signe linguistique est précis pour l'apprenant, et pour cette raison les chances pour que celui-ci opte pour la forme adéquate augmentent considérablement, contrairement aux cas où un adverbe temporel est absent.

Il est donc primordial d'inclure et de souligner dans l'enseignement de la grammaire l'importance des adverbes temporels pour l'identification et l'emploi correct des formes du futur (Noyau 2002). Il s'avère aussi que la position de l'adverbe dans l'énoncé joue un rôle pour le choix des formes du futur. Il est plus facile de choisir la forme du futur appropriée si l'adverbe se trouve en position initiale plutôt qu'en position finale de l'énoncé.

Enfin, il importe de souligner que la terminologie utilisée dans les grammaires n'est pas nécessairement conforme aux valeurs propres des deux formes du futur. Dans certaines grammaires du français langue étrangère, par exemple celle de Hansén et Schwartz (1995, 170), on appelle le futur analytique le futur proche et explique que cette forme décrit une valeur de futur proche du présent, ce qui donne des connotations erronées par rapport à son emploi réel (Franckel 1984). En outre, le terme de futur proche porte sur la sémantique alors que celui du futur simple porte sur la morphologie.

\section{Implications pour l'enseignement}

Afin d'améliorer l'enseignement des formes du futur, il importe au premier abord d'employer une terminologie en accord avec les valeurs propres des formes du futur. On peut 
éviter l'emploi du terme même de futur proche et le remplacer par un terme en accord avec l'usage propre du futur analytique.

L'emploi des adverbes temporels peut aider les apprenants à choisir la forme du futur adéquate, notamment si ceux-là se trouvent en tête de l'énoncé. Les adverbes temporels peuvent donc servir d'outil dans l'enseignement pour rendre plus claire la fonction grammaticale de la forme.

Il faut aussi prendre en considération la langue maternelle de l'apprenant et, par conséquent, le transfert possible entre la langue source et la langue cible. C'est surtout le cas pour l'apprentissage des langues typologiquement hétérogènes comme le danois, langue germanique, et le français, langue romane.

Enfin, dans une optique fonctionnelle et communicative, on peut discuter, selon le niveau des apprenants, s'il faut enseigner des formes qui ne soient pas largement répandues dans la langue quotidienne, et qui posent par conséquent problème aux apprenants. Au niveau universitaire, on ne peut, bien entendu, omettre l'étude approfondie des deux formes du futur, puisqu'il faut fournir un savoir spécialisé aux étudiants à ce niveau éducatif. En revanche, il faudra envisager de ne traiter le futur synthétique que de façon éphémère au niveau secondaire afin de faciliter l'apprentissage en évitant de traiter en profondeur une forme linguistique en réduction, voire en voie de disparition. Il semblerait plus judicieux de mettre l'accent sur les formes les plus répandues pour exprimer le futur, à savoir le futur analytique ou le présent, bien que ce dernier ne soit pas traité dans la présente étude.

\section{Bibliographie}

Andersen, Henning. 2001. "Markedness and the theory of linguistic change." In Actualization. Linguistic Change in Progress, édité par Henning Andersen, 21-57. Amsterdam/Philadelphie: John Benjamins.

Andersen, Henning. 2006. "Grammation, regrammation and degrammation: tense loss in Russian." Diachronica 23, 2: 231-258.

Ayoun, Dalila. 2013. The Second Language Acquisition of French Tense, Aspect, Mood and Modality. Amsterdam/Philadelphia: John Benjamins.

Barceló, Gérard J. et Jacques Bres. 2005. Les temps de l'indicatif en français. Paris: Ophrys.

Confais, Jean-Paul. 1995. Temps, mode, aspect. Les approches des morphèmes verbaux et leurs problèmes à l'exemple du français et de l'allemand. Toulouse: Presses Universitaires du Mirail.

Fleischman, Suzanne. 1982. The Future in Thought and Language. Cambridge: Cambridge University Press.

Franckel, Jean-Jacques. 1984. Futur "simple" et futur "proche". Le français dans le monde 182: 65-70.

Givón, Talmy. 1990. Syntax. A Functional-Typological Introduction. Vol 2. Amsterdam/Philadelphie: John Benjamins.

Gougenheim, Géorges. 1929. Etude sur les périphrases verbales de la langue française. Paris: Les Belles Lettres. 
Hansén, Iah et Björn Schwartz. 1995. La Grammaire. Copenhague: Nordisk Forlag A/S.

Helland, Hans P. 1995. "Futur simple et futur périphrastique: du sens aux emplois." Revue Romane 30, 1: 3-26.

Imbs, Paul. 1968. L'emploi des temps verbaux en français moderne: Essai de grammaire descriptive. Paris: Klincksieck.

Jeanjean, Colette. 1988. "Le futur simple et le futur périphrastique en français parlé. Étude distributionnelle." In Grammaire et histoire de la grammaire. Hommage à la mémoire de Jean Stefanini, édité par Claire Blanche-Benveniste, André Chervel et Maurice Gross, 235-257. Aix-en-Provence: Publication de l'Université de Provence.

Lansari, Laure. 2009. Linguistique contrastive et traduction. Les périphrases verbales aller + infinitif et be going to. Paris: Ophrys.

Larreya, Paul. 2005. "Sur les emplois de la périphrase aller + infinitif." In Les périphrases verbales, édité par Hava Bat-Zeev Shyldkrot et Nicole Le Querler, 337360. Amsterdam/Philadelphie: John Benjamins.

Lindschouw, Jan, 2011. "L'évolution du système du futur du moyen français au français moderne : la réorganisation comme un cas de régrammation", Revue de Linguistique Romane 74: 51-97.

Lindschouw, Jan. 2017. “Tense, aspect, mood." In Manual of Romance Morphosyntax and Syntax, édité par Andreas Dufter et Elisabeth Stark, 397-448. Berlin/Boston: Walter de Gruyter.

Lund, Karen. 2009. "Fokus på sproglæring og pædagogiske implikationer for undervisningen." In Sprogfag i Forandring - Paedagogik og Praksis, édité par Michael Byram, Annette Søndergaard Gregersen, Birgit Henriksen, Anne Holmen, Karen Lund, Merete Olsen et Lars Stenius Stæhr, 127-166. Frederiksberg: Samfundslitteratur.

Noyau, Colette. 2002. "Temporal relations in learner varieties. Grammaticalization and discourse construction." In The L2 Acquisition of Tense-Aspect Morphology, édité par Rafael Salaberry et Yasuhiro Shirai, 107-127. Amsterdam/Philadelphie: John Benjamins.

Nørgård-Sørensen, Jens, Lars Heltoft et Lene Schøsler. 2011. Connecting Grammaticalisation. Amsterdam/Philadelphie: John Benjamins.

Poplack, Shana et Danielle Turpin. 1999. "Does the Futur have a future in (Canadian) French?" Probus 11: 133-164.

Poplack, Shana et Nathalie Dion. 2009. "Prescription vs. Praxis: The Evolution of Future Temporal Reference in French.” Language 85, 3: 557-587.

Reid, T.B.W. 1955. "On the analysis of the tense-system of French." Revue de Linguistique Romane 19: 23-38.

Schrott, Angela 1997. Futurität im Französischen der Gegenwart. Semantik und Pragmatik der Tempora der Zukunft. Tübingen: Narr.

Schrott, Angela 2001. "Le futur périphrastique et l'allure extraordinaire." In Les verbes modaux, édité par Patrick Dendale et Johan van der Auwera, 159-170, Amsterdam/Atlanta: Rodopi.

Togeby, Knud. 1982. Grammaire française. Tome II. Les formes personnelles du verbe. Copenhague: Akademisk Forlag. 
Vet, Co. 1993. "Conditions d'emploi et interprétation des temps futurs du français." Verbum 4: 71-84.

Wiberg, Eva. 2002. "Information structure in dialogic future plans. A study of Italian native speakers and Swedish preadvanced and advanced learners of Italian" In The L2 Acquisition of Tense-Aspect Morphology, édité par Rafael Salaberry et Yasuhiro Shirai, 285-321. Amsterdam/Philadelphie: John Benjamins.

Wilmet, Marc. 1970. Le système de l'indicatif en moyen français. Genève: Droz.

\section{Sources des textes dépouillés}

Frantext. http://atilf.atilf.fr/frantext.htm

Frantext Moyen Français (DMF). http://atilf.atilf.fr/dmf.htm 